\title{
Mitigando la crisis económica del COVID: actuar rápido y hacer lo que sea necesario
}

\section{Mitigating the COVID economic crisis: Act fast and do whatever it takes}

\author{
Baldwin, Richard and Weder di Mauro, Beatrice (eds.) (2020), \\ Mitigating the COVID ECONOMIC CRISIs: ACT FAST AND DO WHATE- \\ ver it TAKes, VoxEU.org eBook, CEPR Press, Londres, 2 I 9 PP., \\ ISBN: 978-I-9I2I79-29-9
}

\section{Introducción}

Este interesante y profuso libro hace un recorrido por las principales decisiones de política económica tomadas -o que, en opinión de los autores, deberían haberse tomado- en distintos países para mitigar los efectos de la pandemia provocada por el COVID-19. A diferencia de la mayoría de los análisis monográficos, que se elaboran una vez que hay suficiente evidencia contrastable sobre el tema en cuestión, la obra analizada apuesta por reunir y presentar de forma descriptiva a la vez que crítica, las respuestas nacionales (principalmente europeas y asiáticas) ante la magnitud y evolución de la emergencia sanitaria, bajo una visión económico-normativa.

La obra reseñada es producto del trabajo colaborativo de un amplio grupo de destacados investigadores en el ámbito económico, liderados por Richard Baldwin y Beatrice Weder di Mauro, profesores de economía internacional en el Instituto de Altos Estudios Internacionales y del Desarrollo (Suiza) y en la Universidad Johannes Gutenberg (Alemania), respectivamente, quienes fungen como editores de ésta. El libro se divide en 24 apartados y un capítulo introductorio firmado por los editores, donde se expone la intención de contribuir al análisis de las reacciones políticas y económicas ante este fenómeno inesperado y cambiante, destacando el riesgo de que malas soluciones temporales puedan acarrear graves problemas duraderos. Esta reseña pretende sintetizar y articular las principales ideas de estos apartados. 


\section{Estructura del libro}

Durante el primer semestre del año 2020 buena parte de los gobiernos del mundo estuvieron trabajando en el diseño e implementación de políticas fiscales, financieras, laborales e industriales, entre otras que, de acuerdo con su visión del problema, reducen el daño económico derivado de la pandemia del COVID-19. Para enfrentar esta coyuntura, Charles Wyplosz sugiere recurrir a los apoyos directos dejando de lado el riesgo moral. Para el autor, la capacidad de los países miembros de la Unión Europea (UE) para coordinarse en esta tarea será un determinante crucial. Aunque la crisis es económica y no financiera, los mercados ya empiezan a entrar en pánico, lo que se observa en la adopción de posiciones cortas por parte de los inversores.

Pierre Olivier Gourinchas propone aplanar la curva epidémica, aunque ello inevitablemente aumentará la curva de recesión que amenaza con dañar la compleja red de vínculos que hacen que la economía funcione. Esta compensación está detrás de las acciones de algunos líderes cuando retrasan las políticas de contención. Para Gourinchas, la prioridad debe ser garantizar el empleo y pago de salarios, y que las empresas puedan evitar la bancarrota, permitiéndoles hacer pagos parciales de impuestos (o condonándoselos) y otorgándoles préstamos en condiciones favorables.

Gita Gopinath plantea que la prioridad debe ser mantener a la gente sana y resguardada. Los cierres comerciales están llevando a la reducción de la producción, sumada a la del consumo, principalmente, de bienes no indispensables. Por otro lado, hay una reducción directa en la oferta de mano de obra (por recortes, licencias forzosas, etcétera). Además, las empresas que dependen de cadenas globales de suministro están dejando de obtener los insumos que necesitan. Para Gopinath, estos efectos serán particularmente graves para sectores como el turismo, el transporte aéreo y la hostelería. En su opinión, el costo del dinero podría aumentar por el temor al incumplimiento de pagos. Una reducción del crédito podría amplificar la desaceleración cuyos efectos se generalizarían a través del comercio internacional y los vínculos financieros. En su opinión, la pandemia tiene a consumidores y a empresas de todo el mundo posponiendo decisiones.

Olivier Blanchard destaca la responsabilidad del Banco Central Europeo en la contención de la crisis y afirma que no debería primar el riesgo moral sino sólo el auxilio para los miembros que lo necesitan, y la consideración de que dicho apoyo podría ser la única vía para salvar la zona. Sin embargo, esto requerirá del acuerdo entre los países miembros.

Alberto Alesina y Francesco Giavazzi se preguntan de qué tamaño debe ser la respuesta política al shock económico provocado por la pandemia. 
Argumentan que la reacción del gobierno italiano fue inicialmente muy modesta, aunque ésta se elevó rápidamente hasta llegar a comprometer un porcentaje del PIB superior al estipulado por las normas de la zona euro. Para los autores, el gobierno tiene que plantearse objetivos claros que den certidumbre a la población, más allá de discusiones presupuestales.

Por otra parte, Jordi Galí indica que la rápida propagación del coronavirus constituye un desafío para los sistemas de salud y que, aun con medidas como el confinamiento, las restricciones de movilidad y el cierre temporal de comercios, no se podrá evitar una importante pérdida de vidas humanas. Esas medidas, aunque necesarias, tendrán un impacto negativo en muchos sectores. Para revertirlo, los gobiernos podrían otorgar a las empresas afectadas (incluyendo a los autoempleados) los recursos para cumplir con sus compromisos ineludibles. Según Galí, el gobierno tiene una alternativa para financiar un programa fiscal de emergencia, a saber: inyectar dinero a la economía a través de los bancos centrales sin registrar activos o pasivos en sus balances. Esta intervención es conocida como 'helicóptero de dinero'.

Stefano Ramelli y Alexander Wagner hablan de la respuesta del mercado de valores a la pandemia. Ellos indican que, al inicio de la emergencia, el mercado mantuvo un buen nivel de retornos, los cuales finalmente se desvanecieron conforme fue creciendo la percepción de que los problemas de liquidez y deuda corporativa podrían llegar a convertirse en una compleja crisis financiera. Para los autores, estas decisiones estuvieron basadas en comportamientos de rebaño y no en expectativas individuales.

Shang Jin Wei cree que esta crisis representa una oportunidad desaprovechada por muchos países. El necesario distanciamiento social también ejercerá un fuerte impacto negativo en el conjunto de la economía, que será crítico para algunos sectores en el medio plazo. Las reservas de efectivo de muchas empresas se agotarán rápidamente, sobre todo en el caso de las micro y pequeñas empresas (MyPEs), que serán las más afectadas. Para Wei, el resultado de la coordinación de los programas de estímulos fiscales entre varios países es de mejor pronóstico que el desarrollo de acciones individuales que podrían terminar afectando sobre todo a los países más pequeños y dependientes del exterior.

Para mantener viva la actividad económica y social, una parte importante del comercio minorista y las tareas académicas y laborales se podrían llevar a cabo en línea, siempre que exista en la sociedad un amplio acceso a internet, aceptación generalizada de pagos digitales y sistemas de entrega económicos y eficientes, de acuerdo con el autor. La crisis está forzando el cambio de hábitos hacia el uso exhaustivo de esta posibilidad tecnológica. El autor asegura que las sociedades que promuevan inversiones a 
favor de la economía digital e identifiquen y aprovechen su potencial, serán los ganadores.

Yi Huang y colaboradores afirman que, durante el período del brote en China, la política económica tuvo como objetivo el apoyo a través de estímulos fiscales, deducciones de impuestos y exenciones de pago de obligaciones a sectores estratégicos e industrias gravemente afectadas por la epidemia, tales como los del transporte, restaurantes, hostelería, manufactura y venta minorista. Ellos refieren que las instituciones financieras implementaron una serie de medidas de apoyo, especialmente orientadas a las MyPEs, entre las que se encuentran la reducción de las tasas de interés, la renegociación de préstamos y el suministro de líneas de crédito a través de una ventanilla exclusiva. Para Huang, el 85\% de las MyPEs no tiene capacidad de sobrevivir más de tres meses a una reducción dramática de la demanda.

Asimismo, los autores encuentran que las plataformas fintech tienen ventajas en comparación con los mecanismos tradicionales de provisión de crédito a las MyPEs, tales como el uso de big data para la calificación crediticia, facilidades en el manejo de requisitos de ingreso, mecanismos para otorgar préstamos a gran escala o la posibilidad de hacer transacciones sin restricciones geográficas. En definitiva, pueden mejorar la tasa de supervivencia de las MyPEs, las que, al mismo tiempo, deben ser incentivadas a mejorar su digitalización.

Jonathan Anderson muestra cómo las prioridades económicas en China fueron cambiando ante la emergencia. Él indica que el crecimiento se había desacelerado a este nivel sólo durante la crisis global 2008-2009 y la caída en las exportaciones de 2014-2015 y que, en ambos casos, se tuvo que dar una respuesta política aguda y dramática, conformada por herramientas macroeconómicas, tales como reducciones en las tasas de interés, inyecciones de liquidez del banco central, emisión de deuda cuasi fiscal y préstamos para proyectos de desarrollo.

Danny Quah presenta la respuesta de política económica de Singapur ante el COVID-19, la cual ha incluido el reembolso de impuestos corporativos, más rápida amortización de las inversiones comprometidas, cofinanciación gubernamental de préstamos de capital de trabajo, flexibilidad en los pagos de alquiler para comercios en propiedades gubernamentales y el rediseño de programas de los sectores de turismo y transporte. Esta respuesta ha tenido tres grandes ejes: el económico, para reparar la caída en la demanda y oferta agregada y fallas de mercado; el científico, para enfrentar los desafíos médicos y de salud, y para generar confianza; y el político, donde, en opinión del autor, las autoridades han demostrado estar al mando de la situación y dispuestas a tomar las decisiones necesarias. 
Inkio Cheong relata que Corea del Sur se destacó por haber desarrollado y aplicado kits de prueba por encima del promedio internacional, lo que al parecer sirvió como medida de contención de contagios. Según Cheong, la industria de Corea del Sur está profundamente integrada con la China, por lo que la interrupción en la producción de piezas y suministros se dejó sentir con fuerza en aquel país. Lo anterior, aunado a las dificultades que presenta la logística internacional, está dejando a muchas corporaciones en una situación complicada. Las políticas de apoyo deberán dirigirse a evitar el cierre de las empresas, por ejemplo: a través de fondos para la conservación del empleo y la desgravación fiscal.

A pesar de ser sólo una medida voluntaria para la mayoría, la población surcoreana decidió quedarse en casa de forma estricta, por lo que muchas MyPEs vieron reducida su demanda al grado de enfrentar la bancarrota. El gobierno estableció un fondo de gestión de emergencias para inyectar liquidez a las MyPEs. Para fomentar el consumo, se enfatizó el apoyo a los hogares de bajos ingresos, cubriendo gastos de subsistencia y otorgando, entre otros, cupones de consumo y subsidios para cuidado de niños. Sin embargo, los criterios de evaluación de los fondos están siendo muy rigurosos, lo que ha dificultado la dispersión.

Agnès Bénassy-Quéré y su equipo plantean que esta grave crisis económica para Europa podría clasificarse en cuatro fases. En la primera, se muestra el efecto en la oferta de algunos sectores productivos, aunque las consecuencias a nivel macro son pequeñas. En la segunda, un fuerte shock de demanda sectorial y regional afecta generalmente al turismo, transporte aéreo, hostelería, comercio y entretenimiento. La tercera fase consiste en una interrupción general aguda de la actividad económica, por un shock de oferta agregada. En la fase cuatro llega la recuperación, que estará en función de la pérdida generalizada de ingresos, quiebras de MyPEs y la histéresis prevalente en los mercados.

Siguiendo a los autores, la respuesta política depende de la fase. Durante la primera, las de mayor efecto son las medidas sectoriales de subvención. En la segunda fase conviene propiciar liquidez a través del aplazamiento del pago de impuestos, contribuciones sociales y programas de subsidios al desempleo. La tercera fase requiere medidas más generalizadas por la necesidad de intensificar el gasto en asistencia, considerando las pérdidas irrecuperables de algunos sectores y la necesidad de apoyo financiero a algunos estratos como el de las MyPEs, lo que puede atenderse a través del crédito y la desgravación fiscal. También puede ser necesario el apoyo a través de transferencias directas. En cuanto a la política monetaria, el mejor curso de acción es una relajación monetaria en coordinación con un estímulo fiscal. La fase cuarta requiere de acciones que reduzcan los efectos de la histéresis. 
Luis Garicano propone que las instituciones europeas ayuden a los Estados miembros a cubrir sus gastos sanitarios. Él se suma a la opinión de que éste es un problema de capacidad fiscal y no de política monetaria. Para garantizar que la economía sobreviva a esta congelación, los miembros deben asegurar a los empleados y MyPEs contra el riesgo de quiebra y estimular la actividad económica, lo cual, según el autor, no está ocurriendo. Garicano cree que se debe contar con un fondo que cumpla con tres objetivos: ayudar a los cubrir los gastos médicos, proporcionar un respaldo financiero a las MyPEs y apoyar los esquemas de protección del empleo. Para el autor, el impacto disruptivo del COVID-19 podría hacer que algunas economías europeas se vean tentadas a renunciar a sus compromisos con el euro ante la posibilidad de un crecimiento descontrolado en su deuda.

Philip Lane señala que, dada la reducción de ingreso tanto en MyPEs como en hogares, asegurar la oferta de crédito puede reducir el tamaño de la crisis. En ese sentido, las subastas de liquidez a tasas muy favorables pueden ayudar siempre que se destinen al sector privado, especialmente a MyPEs de los giros más afectados.

Christian Odendahl y John Springford creen que los efectos de largo plazo sobre la economía pueden ser menos severos que los de la crisis financiera de 2008-2009 en la medida en que los gobiernos actúen con rapidez. La falta de liquidez ha hecho caer los mercados bursátiles y ha desencadenado una huida a la seguridad que se traduce en la revaloración de los bonos gubernamentales. Se requerirá una alianza entre bancos centrales nacionales, banca de inversión, reguladores y secretarías de finanzas que garantice la confianza en el sistema financiero. Además, los gobiernos deberán considerar esquemas de trabajo a corto plazo y apoyo directo a empleados con contratos temporales y autoempleados, que serán de los grupos más afectados, al no estar cubiertos por las medidas de carácter general. Plantean que el pago de un ingreso básico universal (una medida muy controvertida) que sea paulatinamente recuperable vía impuestos, no debe ser descartado como parte de las soluciones.

Los autores también destacan las pérdidas irrecuperables en el sector de servicios. Muchas compras postergadas podrán hacerse una vez terminada la contingencia; los consumidores no podrán, sin embargo, compensar, por ejemplo, los espectáculos cancelados o los viajes no realizados. Así, el consumo social podría ser fuertemente golpeado, sobre todo si las medidas de contención y el miedo continúan por meses.

Ugo Panizza expone que Italia ha presentado un modesto crecimiento del PIB (60\% más bajo que el del promedio de la zona euro en los últimos 25 años). El sector turístico italiano tiene el primer lugar en Europa en términos del personal empleado y el segundo como proporción del PIB. 
Otra característica estructural importante de Italia es que tiene una de las mayores contribuciones europeas, provenientes de las MyPEs, economía informal y trabajadores independientes. Las medidas implementadas por el gobierno italiano incluyen crédito fiscal y disminución de impuestos para los sectores más afectados tales como el turismo y la logística. También, se otorgan para las MyPEs préstamos a largo plazo con tasa cero y suspensión del pago de cuotas de seguridad social y se analiza la implementación temporal del ingreso básico universal que llegue a personas que no estén cubiertas por las medidas de emergencia.

Peter Bofinger y su equipo anticipan que Alemania se verá particularmente afectada por la caída del PIB y comercio mundial debido a su alta dependencia de las exportaciones y la manufactura. Los efectos negativos de las restricciones y medidas de seguridad generarán pérdidas irrecuperables en el consumo social. También afirman que, a corto plazo, el objetivo principal de política económica debe encaminarse a compensar los efectos negativos de la falta de liquidez corporativa y a estabilizar el sistema bancario. En el caso de una desaceleración duradera, la atención debe centrarse en garantizar el ingreso mínimo de las empresas y trabajadores (principalmente aquellos por cuenta propia). El Estado valora también hacer inversiones directas de capital en las empresas, aunque se reconoce que esto sería muy complejo de implementar en las MyPEs.

Thorsten Beck dibuja un escenario en el que ni los hogares son capaces de pagar hipotecas y créditos, ni las empresas de producir bienes o servicios, lo que también socava su capacidad para pagar deudas. Para Beck, si bien parte de esta reducción está impulsada por la demanda, existen también efectos sustanciales de la oferta, llevada por su comportamiento procíclico. Se deben anticipar los efectos negativos de la contracción de los sectores económicos y financieros y actuar contracíclicamente de forma crítica y urgente. De acuerdo con el autor, la respuesta a la emergencia en los países de la UE no ha sido ni homogénea ni coordinada. El autor considera que en la zona euro llegó la hora de estar dispuesto a hacer "lo que sea necesario" para aplanar la curva de recesión y evitar daños duraderos a la economía.

Nora Lustig y Jorge Mariscal afirman que en algunos sectores se ha dado una subestimación de la gravedad del problema que denota falta de preparación y sensibilidad. Por ejemplo, indican que, en las primeras etapas de la pandemia, el gobierno chino ocultó deliberadamente información y silenció a los que manifestaron preocupación en el tema. También, en los Estados Unidos (EUA), la administración Trump hizo un esfuerzo proactivo para minimizar el problema, a la vez que inició tardíamente los esfuerzos de contención. Para Lustig y Mariscal, la percepción sobre la pandemia se construye con base en los conocimientos médicos, 
pero también está en función de las políticas de prevención, detección y contención, y de cómo estas políticas hagan sentir a la población, que cuenta con el respaldo de su gobierno.

A los ojos de Jason Furman, la política ante la pandemia tiene seis premisas: mejor hacer mucho que poco, utilizar los mecanismos existentes, crear nuevos programas cuando sea necesario, no temer a la duplicación de esfuerzos o a que los ganadores sean otros, apoyarse del sector privado tanto como sea posible y asegurarse de que la respuesta sea dinámica y persistente.

Pinelopi Goldberg manifiesta que muchas empresas han cerrado sus instalaciones y exigido a sus empleados que trabajen a distancia y que las videoconferencias están reemplazando a las reuniones presenciales dando lugar a ahorros en tiempo de desplazamiento y a otras externalidades positivas. Al mismo tiempo, los educadores de todos los niveles están trabajando a marchas forzadas en alternativas que reemplacen la instrucción en el aula. Para Goldberg, una vez que los estudiantes puedan regresar a las aulas, se deben seguir aprovechando estas innovaciones, sobre todo en los países en vías de desarrollo. Aunque será necesario asegurarse de compensar a los perdedores y de que el desarrollo económico no incremente la desigualdad social, tratar de retroceder el progreso no debe ser una alternativa.

Adam Posen visualiza al nacionalismo económico como un fenómeno oportunista que puede surgir después de la disminución de la confianza en la economía global y el multilateralismo. Esto también sería en parte el resultado del desarrollo de las agendas políticas de los gobiernos con orientación autárquica que ven en las crisis su gran momento. En opinión del autor, esto no sólo corrompe y desincentiva la inversión y la productividad, sino que también aumenta el riesgo de surgimiento de relaciones de explotación y de conflicto entre naciones. Por otro lado, el desafío económico consiste en articular el financiamiento y la generación de empleo en los sectores de mayor afectación. Muchos de estos sectores están compuestos predominantemente por MyPEs jóvenes, y en algunos casos albergan trabajo informal. La transferencia de préstamos, así como la provisión de crédito comercial y el factoraje financiero tendrían un impacto positivo en esas MyPEs.

Paul Krugman, en el último capítulo, discute la posibilidad de implementar estímulos fiscales y de política monetaria de forma permanente y en combinaciones poco convencionales. En opinión del autor, se debe aceptar que las bajas tasas de interés y el estancamiento secular podrían formar parte de la nueva normalidad a largo plazo. Aunque una política de estímulo permanente puede terminar por elevar las tasas de interés, para Krugman hay factores compensatorios que podrían amortizar el 
riesgo. Bajo una lógica keynesiana, el autor destaca que cuando la economía se encuentra en una trampa de liquidez, la inversión pública adicional tiene un efecto multiplicador. Además, si la inversión es productiva, amplía las capacidades de la economía en materia de infraestructura y de programas de bienestar social. Finalmente, siguiendo al autor, la evidencia de que las recesiones temporales llegan a deprimir la producción, y con ello, el mercado de trabajo (histéresis) también debe tomarse en cuenta.

\section{Conclusiones}

Si no es posible siquiera saber cuánto durará, se antoja realmente complicado poder anticipar las implicaciones que esta pandemia tendrá en el desarrollo de los países y en el bienestar de los ciudadanos. Elementos como el temor social, las nuevas reglas de convivencia, el uso de la tecnología y los cambios en las expectativas de ingreso y de consumo, entre otros, tendrán un peso aún por determinar en la ecuación de lo que se ha dado en llamar nueva normalidad.

De cualquier forma, la obra aquí reseñada es de gran utilidad en un contexto latinoamericano, al menos por dos razones. Por una parte, constituye una herramienta de valuación de la capacidad de reacción y de las decisiones de política económica (en muchos casos sobre la base de prueba y error) de sociedades con diferentes realidades socioeconómicas acerca de un mismo fenómeno coyuntural. Por otra parte, es una referencia para la construcción de las respuestas latinoamericanas al problema, considerando su alta incidencia de trabajadores informales que no es alcanzada por los programas de apoyo convencionales y su mayor dependencia de las remesas internacionales cuyos trabajadores aportantes son justo los más vulnerables en los países donde radican.

José Gabriel Aguilar Barceló gaba@uabc.edu.mx Profesor-investigador de tiempo completo de la Facultad de Economia y Relaciones Internacionales (FEyRI), Universidad Autónoma de Baja California

SANtos López-Leyva sanlop1947@gmail.com

Profesor de la Facultad de Economía y Relaciones Internacionales (FEyRI) Universidad Autónoma de Baja California 
José Gabriel Aguilar Barceló. Doctor en Ciencias Económicas por la Universidad Autónoma de Baja California (UABC), maestro en Finanzas y licenciado en Administración de Empresas por el Instituto Tecnológico y de Estudios Superiores de Monterrey. Profesor-investigador de tiempo completo de la Facultad de Economía y Relaciones Internacionales (FEyRI) de la Universidad Autónoma de Baja California. Integrante del Sistema Nacional de Investigadores, nivel II y de la Academia Mexicana de Ciencias. Sus líneas de investigación se orientan al análisis de la competencia, el desarrollo, la desigualdad, la pobreza y la empresarialidad de base social. Entre sus más recientes publicaciones se encuentran, como coautor: "La expansión de la microempresa informal ¿̨cuestión de desempeño o de empeño?", Contaduría y Administración, 66 (3), Ciudad de México, Universidad Nacional Autónoma de México, pp. 1-22 (2020); como co-coordinador: Innovación social, políticas públicas e instituciones para el desarrollo de las regiones, Tijuana, Universidad Autónoma de Baja California/Ediciones del Lirio (2020); como coautor: "La empleabilidad de los egresados de universidades tecnológicas en México: un análisis de eficiencia”, Revista Iberoamericana de Educación Superior, 10 (29), Ciudad de México, Universia/IISUE de la UNAM, pp. 3-24 (2019).

Santos López-Leyva. Doctor en Economía por la Universidad Nacional Autónoma de México en el área de Economía de la Ciencia y la Tecnología. Profesor de la Facultad de Economía y Relaciones Internacionales de la Universidad Autónoma de Baja California, imparte cursos de Economía de la Educación Superior en el programa de Maestría y Doctorado en Estudios del Desarrollo Global y Doctorado y Maestría en Ciencias Económicas. Integrante del Sistema Nacional de Investigadores nivel III y cuenta con el reconocimiento del Perfil Deseable PRODEP de la Secretaría de Educación Pública. Entre sus más recientes publicaciones se encuentran, como autor: "Fortalezas y debilidades de la educación superior en América Latina para la competitividad global", Formación Universitaria, 13 (5), La Serena, Centro de Información Tecnológica, pp. 165-176 (2020); como coautor: "Las Instituciones de Educación Superior en la conformación de los Sistemas Nacionales de Innovación en los países iberoamericanos", Revista de la Educación Superior, 49 (195), Ciudad de México, Asociación Nacional de Universidades e Instituciones de Educación Superior de la República Mexicana, A.C. (ANUIES), pp. 73-89 (2020), y Escribir la tesis doctoral. Una tarea seductora, Mexicali, Universidad Autónoma de Baja California/Universidad Autónoma de Occidente (2019). 